\title{
Rendimento e composição do óleo essencial de Piper aduncum L. cultivado em Manaus, AM, em função da densidade de plantas e épocas de corte
}

SILVA, A.L. ${ }^{1}$; CHAVES, F.C.M. ${ }^{2 *}$; LAMEIRA, R.C. ${ }^{1}$; BIZZO, H.R. ${ }^{3}$

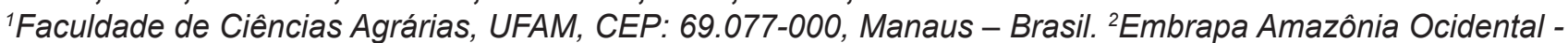

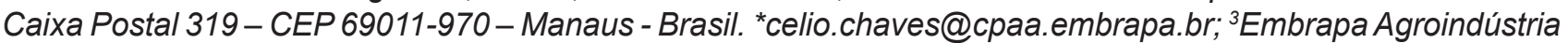
de Alimentos, Av. das Américas, 2950, Bairro Guaratiba, 23020-470 - Rio de Janeiro - Brasil

\begin{abstract}
RESUMO: A pimenta-de-macaco (Piper aduncum L. - Piperaceae) é uma espécie usada na medicina popular em quase todo o Brasil e apresenta alto teor de óleo essencial (2,5 a 4,0\%) rico em dilapiol - éter fenílico com ação inseticida. O presente trabalho teve como objetivo contribuir para o sistema de cultivo da espécie $P$. aduncum nas condições de Manaus AM. O experimento foi realizado na área experimental da Embrapa Amazônia Ocidental. $O$ delineamento utilizado foi o de blocos casualizados com quatro repetições, cada repetição com quatro plantas na área útil. Sete densidades de plantas $(0,5 \mathrm{~m} \times 0,5 \mathrm{~m} ; 1,0 \mathrm{~m} \times 0,5 \mathrm{~m} ; 1,0 \mathrm{~m} \times$ $1,0 \mathrm{~m} ; 1,5 \mathrm{~m} \times 1,0 \mathrm{~m} ; 1,5 \mathrm{~m} \times 1,5 \mathrm{~m} ; 2,0 \mathrm{~m} \times 1,5 \mathrm{~m}$ e 2,0 m x 2,0 m, E1 a E7, respectivamente) e três épocas de corte (C1 - 6 meses após transplantio; $C 2$ - rebroto de $C 1$ aos 6 meses plantas com idade de 12 meses, no campo; C3 - rebroto de C2 aos 6 meses - plantas com 18 meses, no campo), portanto em esquema fatorial. Foi observado que o segundo corte (C2) apresentou maior produção, no espaçamento E4. Não houve diferença estatística para o teor de óleo essencial em $\mathrm{C} 1$, independente da densidade de plantas. Com relação ao rendimento (produção) em óleo essencial, o melhor foi observado com o corte C2, nos espaçamentos E3 e E4, com 76,0 kg/ha e $88 \mathrm{~kg} / \mathrm{ha}$, respectivamente, contendo até $85,6 \%$ de dilapiol no óleo. Assim, a melhor condição de cultivo nas condições de teste foi observada para o período de corte C2 e espaçamentos de 1,0 m x 1,0 m, e de 1,0 m x 1,5 m.
\end{abstract}

Palavras-chave: Piperaceae, planta medicinal, dilapiol, Amazônia.

ABSTRACT: Yield and composition of the essential oil from Piper aduncum L., cultivated in Manaus, AM, regarding plant density and cutting period. Pimenta-de-macaco (monkey pepper - Piper aduncum L. - Piperaceae) is a species used in folk medicine throughout Brazil, containing an essential oil ( 2.5 to $4.0 \%$ yield) rich in dillapiole, an arylpropanoid with insecticide action. The objective of this research was to improve the cultivation system of $P$. aduncum in local conditions of Manaus, AM. The study was conducted in the experimental fields of Embrapa Western Amazon, and used randomized blocks with four replicates, each replicate with four plants in its testing area. Seven plant densities $(0.5 \mathrm{~m} \times 0.5 \mathrm{~m} ; 1.0 \mathrm{~m} \times 0.5 \mathrm{~m} ; 1.0 \mathrm{~m} \times 1.0 \mathrm{~m} ; 1.5$ $\mathrm{m} \times 1.0 \mathrm{~m} ; 1.5 \mathrm{~m} \times 1.5 \mathrm{~m} ; 1.5 \mathrm{~m} 2.0 \mathrm{~m} ; 2.0 \mathrm{~m} \times 2.0 \mathrm{~m}$, E1 to E7 respectively) and three cutting periods (C1 - 6 months after transplanting, C2 - regrowth of C1 after 6 months (plants with 12 months in the field) and C3 - regrowth of C2 after 6 months (plants with 18 months in the field), therefore in a factorial scheme. A larger production was observed in the second cutting (C2) in the plant density E4. There were no statistical differences in the essential oil content in $\mathrm{C} 1$, regardless of densities. In relation to the essential oil yield, the best result was observed for the cutting period C2 in the E3 and E4 densities, $76.0 \mathrm{~kg} / \mathrm{ha}$ and $88 \mathrm{~kg} / \mathrm{ha}$, respectively, with a dillapiole content of up to $85.6 \%$. Therefore, the best cultivation condition according to the test was observed for the cutting after 12 months of transplantation and plant density of $1.0 \mathrm{~m}$ x $1.0 \mathrm{~m}$ or $1.5 \mathrm{~m} \times 1.5 \mathrm{~m}$.

Keywords: Piperaceae, medicinal plant, dillapiole, Amazon.

Recebido para publicação em 21/10/2011 


\section{INTRODUÇÃO}

Piperé o maior gênero da família Piperaceae com mais de 700 espécies, dos quais cerca de 170 crescem de forma nativa no Brasil (Yuncker, 1972). Piper aduncum L. (Piperaceae) é uma planta aromática, nativa da Região Amazônica que apresenta alto teor de óleo essencial (2,5 a $4,0 \%$ ), rico em dilapiol. O dilapiol é um éter fenílico que vem sendo testado com êxito como fungicida, moluscida, acaricida, bactericida e larvicida, com a vantagem de ser um produto biodegradável (Bastos, 1997). No que se refere à tecnologia empregada no cultivo das plantas medicinais, podem existir problemas fitotécnicos usuais como irrigação, fertilidade de solo, ataque de pragas e doenças e, principalmente, as influências ambientais externas que resultam muitas vezes na formação de diversos constituintes químicos em diferentes proporções ou em um reduzido rendimento dos princípios ativos (Bustamante, 1993; Corrêa Jr. et al., 1994). Estes fatores requerem planejamento e controle para obtenção da qualidade e maximização do produto a ser adquirido. Recentemente, a Embrapa Amazônia Ocidental vem desenvolvendo pesquisas com espécies medicinais nativas, com o objetivo de determinar tecnologias de cultivo mais adequadas. Dentre estas, destacam-se várias espécies amazônicas com elevado teor de óleos essenciais, que podem estar na folha (Piperaceae), na madeira (Lauraceae), nas raízes (Cyperaceae), dentre outros órgãos. Várias piperáceas contêm óleo essencial nas suas folhas como, por exemplo: Piper aduncum (pimenta-de-macaco), Piper callosum Ruiz \& Pav. (óleo elétrico), Piper hispidinervum C. DC. (pimentalonga), Piper peltata (L.) Miq. (caapeba), Peperomia pellucida (L.) Kunth. (erva-de-jabuti), dentre outras. Óleos essenciais são utilizados em vários setores das indústrias farmacêutica, química e cosmética. Aperfeiçoar a produção do óleo essencial com a produção agronômica requer estudos de técnicas de propagação, adubação, espaçamentos, época de corte, secagem, dentre outros, sempre associando essa resposta produtiva com o perfil químico destas espécies. Diante do exposto, o presente estudo teve como objetivo contribuir para o sistema de cultivo da espécie Piper aduncum L - Piperaceae, nas condições de Manaus - AM, em função de densidades de plantas e épocas de corte.

\section{MATERIAL E MÉTODOS}

O experimento foi realizado no Campo Experimental da Embrapa Amazônia Ocidental, em Manaus, AM, no período de dezembro de 2006 a junho de 2008. De acordo com a classificação de Köppen, o tipo de clima da região é tropical. O tipo de solo é Latossolo Amarelo Distrófico, com textura muito argilosa, acidez elevada e alto teor de alumínio trocável (Rodrigues, 1972). O solo recebeu calcário na dose de 4 t/ha para correção da acidez, cuja aplicação foi realizada três meses antes, com incorporação mecânica. O delineamento experimental utilizado foi em blocos casualizados com sete tratamentos (E1 - 0,5 m x 0,5 m; E2 - 1,0 $\mathrm{m} \times 0,5 \mathrm{~m}$; E3 - 1,0 m x 1,0 m; E4 - 1,0 m x 1,5 m; E5 - 1,5 m x 1,5 m; E6 - 1,5 m x 2,0 m, E7 - 2,0 m x $2,0 \mathrm{~m}$, correspondendo às seguintes densidades de plantas.há ${ }^{-1}: 40.000 ; 20.000 ; 10.000 ; 6.667 ; 4.444$; 3.333 e 2.500$)$, três épocas de corte (C1 - 6 meses após transplantio; C2 - rebroto de C1 aos 6 meses; C3 - rebroto de C2 aos 6 meses) e quatro repetições em um esquema fatorial de $7 \times 3$. A propagação foi feita a partir de sementes colhidas de plantas de população natural existente na Embrapa Amazônia Ocidental. Após lavagem das espigas maduras, as sementes foram colocadas para secar à sombra durante cinco dias. No dia 19 de setembro de 2006 as sementes foram semeadas em 12 bandejas de poliestireno expandido (128 células), contendo substrato composto de terriço e esterco (2:1), onde cada célula recebeu quatro sementes. Quinze dias após a emergência, foi realizado o desbaste, mantendo-se a planta mais vigorosa. Essas mudas permaneceram em viveiro, recebendo irrigação diária até a data de plantio definitivo no campo. $\mathrm{O}$ transplantio das mudas para o campo foi realizado aos 26 dias (15 de dezembro de 2006), quando as plantas alcançaram a altura média de 10 a $15 \mathrm{~cm}$. Durante os meses de dezembro, janeiro e fevereiro foram realizadas quatro irrigações a intervalos de uma semana devido ao longo período de estiagem. Foram realizadas capinas manuais, de acordo com a necessidade, nos meses de janeiro, abril e junho, assim como após cada época de corte. Os cortes (a $20 \mathrm{~cm}$ acima do solo) foram realizados nos meses de junho e dezembro de 2007 (C1 e C2, respectivamente) e junho de 2008 (C3), correspondendo a 6, 12 e 18 meses de idade, no campo. Para a quantificação da biomassa aérea, as plantas da área útil (considerando efeito de bordadura) foram cortadas e as folhas, caules, inflorescências/infrutescências foram pesadas em separado e duas amostras de $20,0 \mathrm{~g}$ de cada fração foram levadas para estufa tipo circulação de ar, à temperatura de $65^{\circ} \mathrm{C}$ para obtenção da matéria seca. Para a extração do óleo essencial, foram utilizadas $100 \mathrm{~g}$ de folhas frescas das plantas, utilizando-se aparelho tipo Clevenger. Após duas horas de extração, o óleo essencial extraído foi separado e transferido para frascos escuros, lacrados com tampa e batoque e estocados em freezer $\left(\mathrm{a}-10^{\circ} \mathrm{C}\right)$ até o momento da análise da sua composição química. O teor de óleo essencial (em

Rev. Bras. PI. Med., Campinas, v.15, n.4, supl.I, p.670-674, 2013. 
base seca) foi calculado em função do volume de óleo obtido dividido pela massa seca das folhas e o resultado expresso em percentagem. Para o cálculo de produção de óleo essencial, multiplicou-se a produção de folhas obtida pelo teor de óleo em cada parcela. Os dados foram expressos em $\mathrm{kg} / \mathrm{ha}$. As análises da composição química do óleo essencial foram realizadas na Embrapa Agroindústria de Alimentos (Rio de Janeiro -RJ) por cromatografia em fase gasosa acoplada à espectrometria de massas onde utilizou-se o equipamento Agilent $5973 \mathrm{~N}$, equipado com uma coluna capilar HP5MS (5\%-fenil-95\%-metilpolisiloxano, $30 \mathrm{~m} \mathrm{x} \mathrm{0,25} \mathrm{mm} \mathrm{x}$ $0,25 \mu \mathrm{m})$, utilizando hélio $(1,0 \mathrm{~mL} / \mathrm{min})$ como gás carreador. A temperatura do forno foi de 60 a $240^{\circ} \mathrm{Cl}$ min, a $3^{\circ} \mathrm{C} / \mathrm{min}$. e o detector seletivo de massas foi operado no modo ionização eletrônica $(70 \mathrm{eV})$. Foi injetado $1 \mu \mathrm{L}$ de uma solução a $1 \%$ do óleo em diclorometano, com injetor operando a $250^{\circ} \mathrm{C}$ e divisão de fluxo de 1:20. Para a quantificação dos componentes, foram utilizados os valores de área (\%) obtidos com o uso de um detector de ionização por chama. Para a identificação, os espectros de massas e os índices de retenção obtidos foram comparados com aqueles da espectroteca Wiley 6th ed. e com valores da literatura, respectivamente. Os dados foram submetidos à análise de variância e teste de médias (Tukey a $5 \%$ ) e as variáveisrespostas, quando a interação foi significativa, estão apresentadas em equações de regressão. Durante todo o experimento, foram coletadas, mensalmente, as variáveis climáticas referentes à precipitação pluviométrica $(\mathrm{mm})$ e insolação $(\mathrm{h})$, baseadas nos dados do Laboratório de Agroclimatologia da Embrapa Amazônia Ocidental (Figura 1).

\section{RESULTADOS E DISCUSSÃO}

A Figura 2 mostra os resultados para produção de folhas onde se observou que os maiores valores foram verificados para o C2 até o E4. Para o C1, a partir do E1, houve redução contínua na produção de folhas. Já o C3 demonstrou resposta decrescente, contrária aos demais cortes, apesar de apresentar valores inferiores, principalmente, nos cinco arranjos iniciais. No entanto, em E7, as produções obtidas em C3 foram superiores aos demais tratamentos. Embora visualmente os maiores diâmetros tenham sido observados nos dois maiores espaçamentos (E6 e E7), provavelmente, as reservas acumuladas nas raízes não foram suficientes para proporcionar a regeneração/rebroto, pois houve corte a cada seis meses. Além disso, foi observado que as maiores precipitações e menores valores de horas de luz foram registrados nos seis primeiros meses de cada ano (Figura 1). Para o teor de óleo essencial, não houve interação significativa para os tratamentos quando foi realizado o primeiro corte (C1). O maior teor de óleo essencial foi por ocasião do C2, decrescendo a partir do E5. Para o C3, o teor não tende a cair mesmo com o aumento do espaçamento (Figura 3). Associando-se as condições climáticas de precipitação pluviométrica por ocasião do segundo corte, realizado em dezembro de 2007, denota-se pela Figura 1, que o período anterior de desenvolvimento correspondeu à alta precipitação, alta umidade, fatores importantes para o desenvolvimento dos vegetais.

Para o C3, a curva apresentou um ligeiro aumento até o E3, decrescendo a partir deste. Segundo Campos \& Canéchio Filho (1975), vários fatores podem causar a variação na porcentagem de óleo obtido, destacando-se a idade da planta, tipo de solo cultivado e a técnica de extração empregada. Para a variável de produção de óleo essencial, o C2 foi superior aos demais cortes, mostrando uma alta produção no E4, o que pode ser constatado na Figura 4. Santos \& Innecco (2004), em experimento com Lippia alba (Mill) onde foram realizadas apenas duas colheitas, aos 60 e aos 120 dias, observaram que esta última proporcionou o maior rendimento de óleo. Segundo os autores, tal resultado ocorreu pelo fato da planta estar plenamente estabelecida na segunda colheita. A menor produção de óleo essencial foi verificada em C3, mesmo sendo crescente nos maiores espaçamentos, sendo superior aos demais no E7. C1 e C2 mostraram uma tendência de queda conforme o aumento dos espaçamentos, especialmente quando maiores que E5. Esse decréscimo pode estar associado à redução da precipitação e dos níveis de incidência de radiação solar no período do C3 que podem ter induzido uma queda nos níveis do metabolismo primário e secundário das plantas (Taiz \& Zeiger, 1998). Acrescente-se o fato de que a produção de folhas também foi decrescente e contínua, influenciando, por conseguinte a produção, visto que esta resulta do teor versus produção de folhas. Segundo Choudhury (1994), Fátima et al. (1999), Leal et al. (2001) e Taveira (2003), as épocas de corte, principalmente no que se refere às condições edafoclimáticas do ano, interferem na produção de óleo essencial. O óleo essencial de folhas de $P$. aduncum apresentou os seguintes constituintes: miristicina, beta-cariofileno, cisocimeno, trans-ocimeno, mirceno e dilapiol, sendo este último, o componente majoritário desta população, com teores acima de $80 \%$, ficando os demais em torno de 1 a 2,4\%. Enquanto o teor de dilapiol foi menor no E7, os demais constituintes, com exceção para mirceno, foram superiores exatamente neste espaçamento (E7) (Tabela 1). O dilapiol aparece como constituinte principal no

Rev. Bras. PI. Med., Campinas, v.15, n.4, supl.I, p.670-674, 2013. 
TABELA 1. Valores médios dos constituintes químicos do óleo essencial de pimenta-de-macaco (Piper aduncum L.) em função de espaçamentos e épocas de corte. Manaus - AM, 2009.

\begin{tabular}{|c|c|c|c|c|c|}
\hline Espaçamentos & Dilapiol & Mirceno & Cis-ocimeno & Beta-cariofileno & Miristicina \\
\hline $\mathrm{E} 1$ & $85,17 a b$ & 1,29 & $0,80 \quad c$ & 1,95 & $2,09 \mathrm{a}$ \\
\hline E2 & $85,84 a b$ & 1,19 & 0,82 bc & 1,90 & $1,90 a b$ \\
\hline E3 & 86,13 a & 1,19 & 0,88 bc & 1,80 & $1,89 a b$ \\
\hline E4 & $85,18 a b$ & 1,29 & $0,93 a b$ & 1,85 & $1,94 a b$ \\
\hline E5 & $85,77 a b$ & 1,20 & $0,90 \mathrm{abc}$ & 1,70 & $1,67 \quad b$ \\
\hline E6 & $85,50 a b$ & 1,13 & $0,90 \mathrm{abc}$ & 1,98 & $1,92 a b$ \\
\hline E7 & $83,98 \quad b$ & 1,24 & $1,01 \mathrm{a}$ & 2,20 & $2,17 \mathrm{a}$ \\
\hline C.V. (\%) & 1,60 & 11,93 & 8,83 & 19,39 & 13,71 \\
\hline D.M.S. & 2,00 & 0,21n.s. & 0,11 & 0,54 n.s. & 0,39 \\
\hline Épocas de Corte & Dilapiol & Mirceno & Cis-ocimeno & Beta-Cariofileno & Miristicina \\
\hline $\mathrm{C} 1$ & 85,29 & 1,30 & $1,00 \mathrm{a}$ & $1,64 \mathrm{~b}$ & $1,86 \quad b$ \\
\hline $\mathrm{C} 2$ & 85,57 & 1,14 & $0,72 b$ & $2,37 \mathrm{a}$ & $2,08 \mathrm{a}$ \\
\hline C3 & 85,23 & 1,21 & $0,95 a$ & $1,73 b$ & $1,88 a b$ \\
\hline C.V. (\%) & 1,60 & 11,93 & 8,83 & 0,54 & 13,17 \\
\hline D.M.S. & 1,03n.s. & 0,10 n.s. & 0,06 & 0,28 & 0,19 \\
\hline
\end{tabular}

n.s = Não significativo ao nível de $5 \%$ de probabilidade. Médias seguidas de mesma letra na coluna, não diferem entre si pelo Teste de Tukey a $5 \%$ de probabilidade. $(E 1=0,5 \times 0,5 \mathrm{~m} ; \mathrm{E} 2=0,5 \times 1,0 \mathrm{~m} ; \mathrm{E} 3=1,0 \times 1,0 \mathrm{~m}$; E4 = 1,0 × 1,5 m; E5 = 1,5 x 1,5 m; E6 = 1,5 × 2,0 m; $\mathrm{E} 7=2,0 \times 2,0 \mathrm{~m})$. Cortes: Cortes: $\mathrm{C} 1=6$ meses; $\mathrm{C} 2=$ rebroto de $\mathrm{C} 1 ; \mathrm{C} 3=$ rebroto de $\mathrm{C} 2$.

óleo essencial de $P$. aduncum variando de $58 \%$ a 88,4\% (Smith \& Kassim, 1979; Gottlieb et al., 1981).

Variação no teor dos princípios ativos influenciado pela época de corte das plantas medicinais e aromáticas foi encontrada por diversos autores como Mattos (2000) em alecrim-pimenta (Lippia sidoides Cham.) e Blanco (2001) com alecrim (Rosmarinus officinalis L.). No entanto, nota-se que os diferentes espaçamentos e épocas de corte avaliados neste estudo, não interferiram na qualidade do óleo essencial em relação ao seu constituinte majoritário. Portanto, para a maior obtenção de óleo essencial de $P$. aduncum $L$. e consequentemente na porcentagem de dilapiol, o corte deverá ocorrer aos 12 meses de idade da planta, com espaçamento entre $1,0 \mathrm{~m} \times 1,0 \mathrm{~m}$ a 1,0 $\mathrm{m} \times 1,5 \mathrm{~m}$.

\section{AGRADECIMENTOS}

Ao conselho Nacional de Desenvolvimento Cientifico e Tecnológico (CNPq) pela concessão de bolsa e à Embrapa Amazônia Ocidental.

\section{REFERÊNCIA}

BASTOS, C.N. Efeito do óleo de Piper aduncum sobre Crinipellis perniciosa e outros fungos fitopatogênicos. Fitopatologia Brasileira, v.3, n.22, p.441-443, 1997.

BLANCO, M.C.S.G. Influência de épocas de colheita na produção de óleo essencial de alecrim. In: JORNADA PAULISTA DE PLANTAS MEDICINAIS, 5. Anais... p.74, 2001.

BUSTAMANTE, F.M.L. Plantas medicinales y aromaticas. Madrid: Mundi Prensa, 1993, p.85-88.

CAMPOS, T.; CANÉCHIO FILHO, V. Principais culturas. 2ed. Campinas: Instituto Campeiro de Ensino Agrícola v. $02,1975.633 p$.

CHOUDHURY, S.N. Effect of clipping heigth on herb and essential oil yield of lemongrass (Cymbopogon flexuosus). Indian Journal Agronomy, v. 39, n. 4, p. 592-598, 1994.

CORRÊA JÚNIOR, C.; MING, L.C.; SCHEFFER, M.C. Cultivo de plantas medicinais, condimentares e aromáticas, 2. ed., Jaboticabal: FUNEP, p. 162, 1994. FÁTIMA, S.; FAROOQI, A.H.A.; ANSARI, S.R.; SRIKANT, S. Effect of water stress on growth and essential oil metabolism in Cymbopogon martini (palmarosa) cultivars. Journal of Essential Oil Research. v. 11, n.4, p. 491-496, 1999.

GOTTLIEB, O.R.; KOKETSU, M.K.; MAGALHÃES, M.T.; MAIA, J.G.S.; MENDES, P.H.; ROCHA, A.I.; SILVA, M.L.; WILBERG, V.C. Óleos essenciais da Amazônia. VII. Acta Amazonica, 11 (1): 143-148. 1981.

LEAL, T.C.A.B.; FREITAS, S.P.; SILVA, J.F.; CARVALHO, A.J.C. Avaliação do efeito da variação estacional e horário de colheita sobre o teor foliar de óleo essencial de capim cidreira [Cymbopogon citratus (DC) Stapf]. Revista Ceres, v.48, n.278, p. 445-453, 2001.

MATTOS, S.H. Estudos fitotécnicos da Mentha arvensis L. var. Holmes como produtora de mentol no Ceará. 2000. 98p. Tese (Doutorado UFC/CCA), Fortaleza.

RODRIGUES, T.E. Levantamento detalhado dos solos do IPEAOC, MA-DNPEA-IPEAOC. Manaus, 63 p. (Boletim técnico). 1972.

SANTOS, M.R.A.; INNECCO, R. Adubação orgânica e altura de corte da erva-cidreira brasileira. Horticultura Brasileira, v.22, n.2, p.182-5, 2004.

SMITH, R.M.; KASSIM, H. The essencial oil of Piper aduncum from Fiji. New Zeland Journal Science. 22: 
127-128. 1979.

TAIZ, L; ZEIGER, E. Plant physiology. 2. ed. Sunderland Massachusetts: Sinaver Associates, 1998. 792p.

TAVEIRA, F.S.N.; ANDRADE, E.H.A.; LIMA, W.N.; MAIA, J.G.S. Seasonal variation in the essential oil of
Pilocarpus microphyllus Stapf. Anais da Academia Brasileira de Ciências. vol.75 n.1 Rio de Janeiro Mar. 2003.

YUNCKER, T.G. The Piperaceae of Brazil. Hoehnea. 2:19-366. 1972. 